\title{
Neonicotinoids disrupt memory, circadian behaviour and sleep
}

\author{
Kiah Tasman ${ }^{1}$, Sergio Hidalgo ${ }^{1}$, Bangfu Zhu ${ }^{1}$, Sean A. Rands ${ }^{2}$ \& James J. L. Hodge ${ }^{1 \bowtie}$
}

Globally, neonicotinoids are the most used insecticides, despite their well-documented sublethal effects on beneficial insects. Neonicotinoids are nicotinic acetylcholine receptor agonists. Memory, circadian rhythmicity and sleep are essential for efficient foraging and pollination and require nicotinic acetylcholine receptor signalling. The effect of field-relevant concentrations of the European Union-banned neonicotinoids: imidacloprid, clothianidin, thiamethoxam and thiacloprid were tested on Drosophila memory, circadian rhythms and sleep. Field-relevant concentrations of imidacloprid, clothianidin and thiamethoxam disrupted learning, behavioural rhythmicity and sleep whilst thiacloprid exposure only affected sleep. Exposure to imidacloprid and clothianidin prevented the day/night remodelling and accumulation of pigment dispersing factor (PDF) neuropeptide in the dorsal terminals of clock neurons. Knockdown of the neonicotinoid susceptible D $\alpha 1$ and $D \beta 2$ nicotinic acetylcholine receptor subunits in the mushroom bodies or clock neurons recapitulated the neonicotinoid like deficits in memory or sleep/circadian behaviour respectively. Disruption of learning, circadian rhythmicity and sleep are likely to have far-reaching detrimental effects on beneficial insects in the field.

An estimated $84 \%$ of European crops are dependent on pollinators whose services are valued at $>€ 22$ bn/year and are essential to food security ${ }^{1,2}$. Globally, $35 \%$ of crop production relies on pollination and these services are valued at $€ 153 \mathrm{bn} /$ year $^{3,4}$. However, populations of pollinating insects are declining dramatically. For instance flying insects have decreased by over 75\% in Germany over the last 27 years ${ }^{5}$. Diminishing pollinator numbers are a serious threat to our food security ${ }^{2,6}$, with intensive use of insecticides being implicated in these losses ${ }^{2,6}$. However, a third of the global crop is lost to pests and without pesticides this loss could be $75 \%$, keeping the demand for insecticides high ${ }^{7,8}$. The most common insecticides worldwide are neonicotinoids, which account for $24 \%$ of the global insecticide market valued at $\$ 1$ billion/year ${ }^{8,9}$. Neonicotinoids are highly efficacious insecticides however they lack specificity, affecting both target pest species such as aphids and non-target beneficial insects, such as bees. They share a mechanism of action, being agonists of nicotinic acetylcholine receptors (nAChR), the main neurotransmitter in the insect nervous system. They also display target site cross-resistance in pests, diminishing their effectiveness as insecticides and unfortunately encouraging application of increasing concentrations ${ }^{9,10}$. They were branded safe compared to previous insecticides and because they have little effect on mammalian nAChRs ${ }^{9,10}$. However, few precursive safety tests were performed/published on beneficial insects, for which neonicotinoids are now known to be potent neurotoxins with well-documented lethal and sub-lethal effects ${ }^{1,7,9,11}$. Therefore, continued intensive use is likely to have severe consequences on insect species numbers, with knock-on as well as direct effects on the ecosystem, aquatic life, birds and mammals including potential toxicity to humans ${ }^{1,12-14}$. This has resulted in the European Union (EU) banning the nitrome neonicotinoids; imidacloprid, clothianidin and thiamethoxam (a prodrug for clothiandin ${ }^{15}$ ) in 2017 followed by the cyanoimine neonicotinoid, thiacloprid in $2020^{16}$. Despite this the neonicotinoids remain the most widely used class of insecticide globally and a number of studies show there has been no decrease in the quantity of banned neonicotinoids found in different populations of honey and bumble bee across Europe a year after the ban ${ }^{17,18}$. Furthermore, some national governments have granted multiple exemptions for the spraying of oil seed rape and a number of other applications ${ }^{19}$. Furthermore neonicotinoids have high solubility and persistence in the environment ${ }^{1}$. Therefore, despite the current EU ban, insects are still at risk of neonicotinoid exposure.

In the field, concentrations of neonicotinoids encountered by non-target insects are typically between 1 and $51 \mu \mathrm{g} / \mathrm{L}$ for seed treated crops and 61-127 $\mu \mathrm{g} / \mathrm{L}$ for sprayed crops ${ }^{11}$. Concentrations as low as $1 \mu \mathrm{g} / \mathrm{L}$ (or 1 part per billion (ppb)) can cause significant behavioural effects due to the high potency of neonicotinoids, these include reduced foraging motivation ${ }^{20}$ and circadian rhythms $\mathrm{s}^{21}$ in the bumblebee Bombus terrestris ${ }^{20}$. The potential sub-lethal effects of neonicotinoids are very far-reaching because of the central role of nAChR in synaptic

${ }^{1}$ School of Physiology, Pharmacology and Neuroscience, University of Bristol, Biomedical Sciences Building, University Walk, Bristol BS8 1TD, UK. '2School of Biological Sciences, University of Bristol, Life Sciences Building, Tyndall Avenue, Bristol BS8 1TQ, UK. ${ }^{\circledR}$ email: james.hodge@bristol.ac.uk 
neurotransmission in the insect brain ${ }^{9,10}$. Neonicotinoids cause this ligand-gated ion channel to open, thereby depolarising the neuron and increasing excitability. Prolonged exposure to the depolarising agonist may result in depolarising block, where the protracted depolarisation causes inactivation of the voltage sensitive $\mathrm{Na}^{+}$channels required for action potential firing and the long-term agonist exposure causes nAChR desensitization ${ }^{10,22}$. These effects are likely to have pronounced effects on memory formation and consolidation required for effective foraging in many pollinating insects.

Previous research in Drosophila demonstrated that both the Kenyon cells (which constitute the insect memory centre called the mushroom body ${ }^{23}$ ) and their output neurons (which mediate memory valence) are nicotinic ${ }^{24}$. The mushroom body is also involved in sleep in insects ${ }^{25}$. In honeybees, sub-lethal neonicotinoids electrically inactivated ${ }^{22}$ and decreased the synaptic density ${ }^{26}$ of mushroom body neurons and resulted in disrupted olfactory memory ${ }^{22}$. In bumblebees, exposure during development reduced mushroom body growth, causing learning deficits in adults ${ }^{27}$. Neonicotinoids also reduced honeybee antennal lobe $\mathrm{Ca}^{2+}$ responses and caused sensory deficits $^{28}$, potentially contributing to olfactory memory deficits.

Memory formation is also reliant on circadian rhythms $s^{29,30}$ and sleep ${ }^{31,32}$. Work in honeybees and bumblebees has shown that neonicotinioids can reduce behavioural rhythmicity and disrupt sleep behaviour in these important pollinators ${ }^{21,33}$. The mode of action of neonicotinoids on circadian rhythmicity and sleep is unknown. However, work in Drosophila has shown nAChR signalling is required for setting of the central clock and communication between the light sensing organs and the central clock ${ }^{34-37}$. The timing of sleep/wake cycles is also determined by the circadian clock $^{38}$ with the key clock neurons that mediate arousal expressing nAChRs ${ }^{35,39}$.

The pacemaker neurons of the insect clock consist of the pigment dispersing factor (PDF) neuropeptide expressing small and large ventral lateral neurons (s- and l-LNvs). The s-LNvs maintain rhythmicity in constant conditions and set the pace of the insect clock via PDF signalling ${ }^{40}$. The LNvs express both nicotinic and muscarinic acetylcholine receptors ${ }^{39,41,42}$. They receive ACh from the visual circuit including the lamina, with the s-LNvs also receiving ACh-mediated light input information from the Hofbauer-Buchner (HB) eyelets ${ }^{34,35}$. These excitatory signals regulate the electrical excitability of the LNvs which is required for circadian function ${ }^{43}$, with the neurons being more depolarised and having increased firing rate in the day than at night ${ }^{44}$. These day/ night differences in excitability help sustain the molecular oscillation of clock genes in constant conditions as well as regulating day/night changes in s-LNv terminal remodelling and PDF release necessary for robust behavioural rhythmicity ${ }^{43}$. The s-LNv dorsal terminals exhibit circadian remodelling, with their terminals being more branched during the day than at night ${ }^{45}$ and having higher PDF accumulation in the day than at night ${ }^{43,46}$. $\mathrm{LNv}$ expression of a GCaMP $\mathrm{Ca}^{2+}$ reporter showed that $4 \mu \mathrm{g} /$ l clothiandin caused a transient $(\sim 1 \mathrm{~min})$ increase in $\mathrm{Ca}^{2+}$ dependent fluorescence ${ }^{33}$. While similar experiments using a nAChR promoter Gal4 line (which drives expression wherever the endogenous $\mathrm{nAChR}$ is expressed) to express GCaMP showed that $2.5 \mu \mathrm{g} / \mathrm{L}$ imidacloprid blocked nAChR agonist (e.g. carbachol) evoked $\mathrm{Ca}^{2+}$ responses in larval neurons. A 2 h exposure of $2.5 \mu \mathrm{g} / \mathrm{L}$ imidacloprid increased gut and brain levels of superoxide reactive oxygen species and decreased mitochondrial function and ATP levels ${ }^{47}$. Chronic exposure of $4 \mu \mathrm{g} / \mathrm{L}$ imidacloprid led to degeneration of the fly retinae and loss of visual acuity, providing further evidence that field relevant sublethal concentrations of neonicotinoids bring about irreversible effects at the neuronal level.

The circuitry and molecular components of the mushroom body and the clock identified in Drosophila are shown to be highly conserved amongst insects ${ }^{25,48,49}$ making it a powerful model to test the effects of neonicotinoids on memory, circadian behaviour and sleep. Drosophila have ten different nAChR subunits most of which are highly conserved across insect species, making it probable that a neurotoxin selected for its high potency to target insect $\mathrm{nAChRs}$ will affect the equivalent $\mathrm{nAChR}$ in beneficial insects ${ }^{50}$. Whilst the subunit conformation and location of neonicotinoid susceptible nAChRs is still largely unknown, in Drosophila the subunits Da1 and $D \beta 2$ have been shown to play a role in neonicotinoid susceptibility and resistance, with mutations in these conveying both neonicotinoid resistance and significant fitness costs ${ }^{9,10,51}$. Given the power of Drosophila as a model system, and the likely generalisation provided by conservation of nAChR function across insects, we tested the sub-lethal effect of field-relevant concentrations of four major neonicotinoids on Drosophila memory, circadian rhythms and sleep.

\section{Results}

Neonicotinoids reduce longevity, offspring viability and climbing ability in Drosophila. Field relevant concentrations of neonicotinoids cause a range of lethal and sub-lethal effects in bees ${ }^{1,52}$. In order to validate the use of Drosophila as a model for these lethal and sub-lethal effects, we fed field relevant concentrations of four commonly used neonicotinoids to Drosophila and determined their effect on longevity, offspring viability and climbing ability. As in pollinators, longevity, fecundity and mobility were all affected by neonicotinoid exposure in Drosophila ${ }^{1,53-55}$. The mean lifespan of control flies was 49 days while exposure to a field relevant concentration of $10 \mu \mathrm{g} / \mathrm{L}$ clothianidin causing a reduction to 28 days, imidacloprid and thiamethoxam to 36 days, and thiacloprid, to 39 days (Supplementary Fig. S1). The number of eggs laid were increased by 1 or $10 \mu \mathrm{g} / \mathrm{L}$ of thiamethoxam or clothianidin or $100 \mu \mathrm{g} / \mathrm{L}$ imidacloprid (Supplementary Fig. S2). However, the overall viability of the eggs was reduced, with $100 \mu \mathrm{g} / \mathrm{L}$ clothianidin, thiamethoxam or thiacloprid and 10 or $100 \mu \mathrm{g} / \mathrm{L}$ imidacloprid reducing the actual proportion of eggs that subsequently completed development and successfully eclosed as adults (Supplementary Fig. S3). Likewise, field relevant concentrations of imidacloprid, clothianidin and thiamethoxam $(10$ and $50 \mu \mathrm{g} / \mathrm{L})$ all reduced locomotor performance, tested via a negative geotaxis climbing assay, whilst thiacloprid had no effect on locomotion at these concentrations (Supplementary Fig. S4).

Neonicotinoids reduced memory in Drosophila. Olfactory associative memory is critical for foraging pollinators and has been shown to be disrupted by neonicotinoids in bees ${ }^{56}$. In order to see if field relevant 


\section{a}
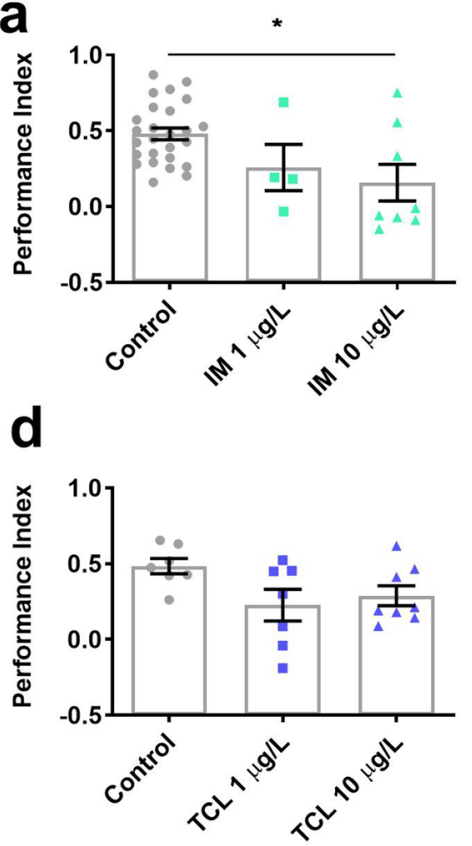

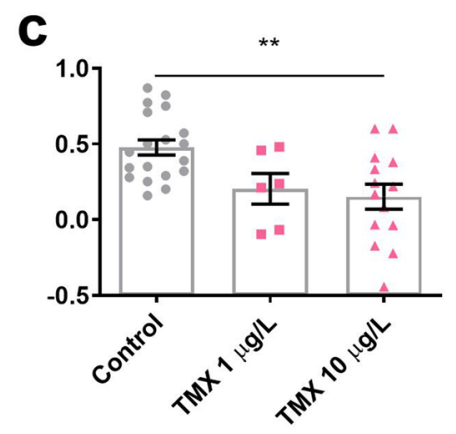

e
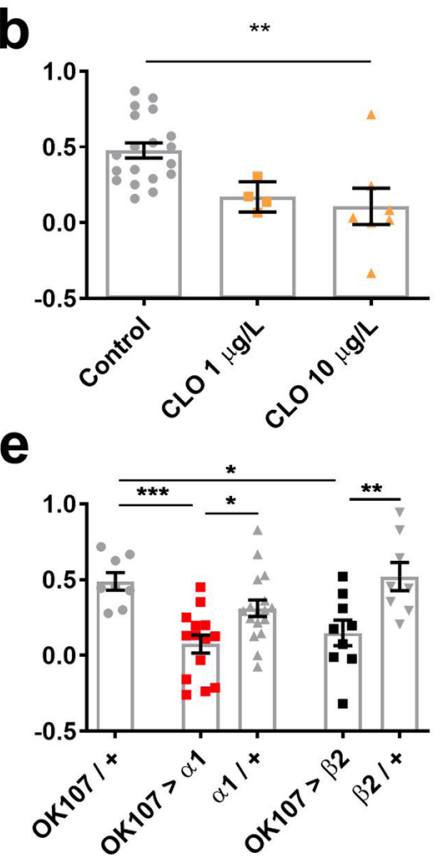

Figure 1. Field relevant concentrations of neonicotinoids or knockdown of Da1 or D $\beta 2$ in the mushroom bodies reduced $1 \mathrm{~h}$ memory. $1 \mathrm{~h}$ memory was reduced in flies exposed to field relevant concentrations of 1 or $10 \mu \mathrm{g} / \mathrm{L}$ (a) imidacloprid (IM) $\left.\left(\chi_{2}^{2}\right)=7.3, p=0.026\right)$, (b) clothianidin (CLO) $\left(\chi^{2}{ }_{2}=12.4, p=0.002\right)$, (c) thiamethoxam (TMX) $\left(\chi_{2}^{2}=9.6, p=0.008\right)$ and not in (d) thiacloprid (TCL) $\left(\chi_{2}^{2}=5.0, p=0.084\right)$. (e) Likewise, $1 \mathrm{~h}$ memory was reduced in flies with RNAi mediated knockdown of Da1 (OK107-Gal4> uas-nAChR-D $\alpha 1)$ or $\mathrm{D} \beta 2$ (OK107-Gal4 > uas- $n A C h R-D \beta 2)$ throughout the mushroom body $\left(F_{2,20}=4.6, p=0.023\right)$ and compared to genotype controls. Each data point represents $\sim 100$ flies, $\mathrm{n} \geq 4$ per treatment. Graphs show mean \pm standard error of the mean (SEM) (post hoc pairwise comparisons: $p \leq 0.05^{\star}, p \leq 0.01^{\star *}, p \leq 0.001^{* * *}, p \leq 0.0001^{* * *}$ ). The same tests, error bars and $p$ values were used throughout.

concentrations of imidacloprid, clothianidin, thiamethoxam and thiacloprid had a similar effect on flies, $1 \mathrm{~h}$ memory (Fig. 1) was assessed using Drosophila olfactory shock conditioning ${ }^{57}$. Imidacloprid, clothianidin and thiamethoxam all reduced memory at $10 \mu \mathrm{g} / \mathrm{L}$ (Fig. 1a-c) whilst thiacloprid left memory intact (Fig. 1d). Sensory controls showed that none of the neonicotinoids tested reduced the ability of flies to sense either the odours or the aversive stimuli (Supplementary Fig. S5). In order to localise the effect of nAChR mis-regulation on memory, we expressed RNAi transgenes to the neonicotinoid sensitive nAChR Da1 and D $\beta 2$ subunits, which had previously been shown to block nAChR mediated $\mathrm{Ca}^{2+}$ responses in the mushroom body memory circuit ${ }^{24}$. In order to further validate the RNAi lines we performed qRT-PCR on brains extracted from flies that pan-neuronally expressed (with elav-Gal4) the Da1 or D $\beta 2$ transgenes and measured their $D \alpha 1$ or $D \beta 2$ mRNA expression which was shown to be significantly decreased by $33 \%$ and $58 \%$, respectively (Supplementary Fig. S10). Knockdown of $\mathrm{D} \alpha 1$ and $\mathrm{D} \beta 2$ throughout the mushroom body significantly reduced memory to a similar level to that caused by neonicotinoid exposure (Fig. 1e), confirming the importance of these subunits in mushroom body mediated memory and the effect of neonicotinoids on this behaviour.

Neonicotinoids decrease circadian rhythmicity and sleep in Drosophila. The effect of neonicotinoids on circadian rhythmicity and sleep was then measured as these behaviours are known to be regulated by nAChR signalling $25,35,36,39$. The effect of field relevant concentrations of neonicotinoids on circadian rhythms was tested using the Drosophila Activity Monitor (DAM2, Trikinetics Inc, USA) ${ }^{58}$. Imidacloprid, clothianidin and thiamethoxam all reduced circadian rhythmicity (Fig. 2), with flies showing greatest sensitivity to thiamethoxam, which caused a reduction in mean rhythmicity at 1,10 and $50 \mu \mathrm{g} / \mathrm{L}$ (Fig. 2d) while clothianidin and imidacloprid caused a reduction in mean rhythmicity at $50 \mu \mathrm{g} / \mathrm{L}$ (Fig. 2b,c). Any concentration of the three neonicotinoids caused an increase in the proportion of flies that were arrhythmic (rhythmicity statistic (R.S.) $\leq 1.5)$ compared to controls (Fig. 2f-i, Supplementary Table S1). Again, thiacloprid appeared not to have sub-lethal effects, with field-relevant concentrations leaving circadian rhythmicity intact (Fig. 2e,i). None of the neonicotinoids affected the free-running period length (Supplementary Fig. S6). Under LD conditions, the effects on rhythmicity were much smaller, with RS remaining high and only IM and TMX having an effect (Supplementary Fig. S7).

Sleep was also monitored using the DAM system, with bouts of inactivity lasting more than 5 min qualifying as sleep ${ }^{37}$. Field relevant concentrations of all four neonicotinoids caused fragmentation of sleep, arising from sleep formed of a greater number of sleep episodes (Fig. 3e-h) of shorter length compared to control (Fig. 3i-l). This effect was greatest for clothianidin, where 1,10 and $50 \mu \mathrm{g} / \mathrm{L}$ caused fragmentation of both daytime and nighttime sleep (Fig. 3f,j) resulting in a reduction of night-time sleep (Fig. 3b, Supplementary Fig. S8). Thiamethoxam 
a
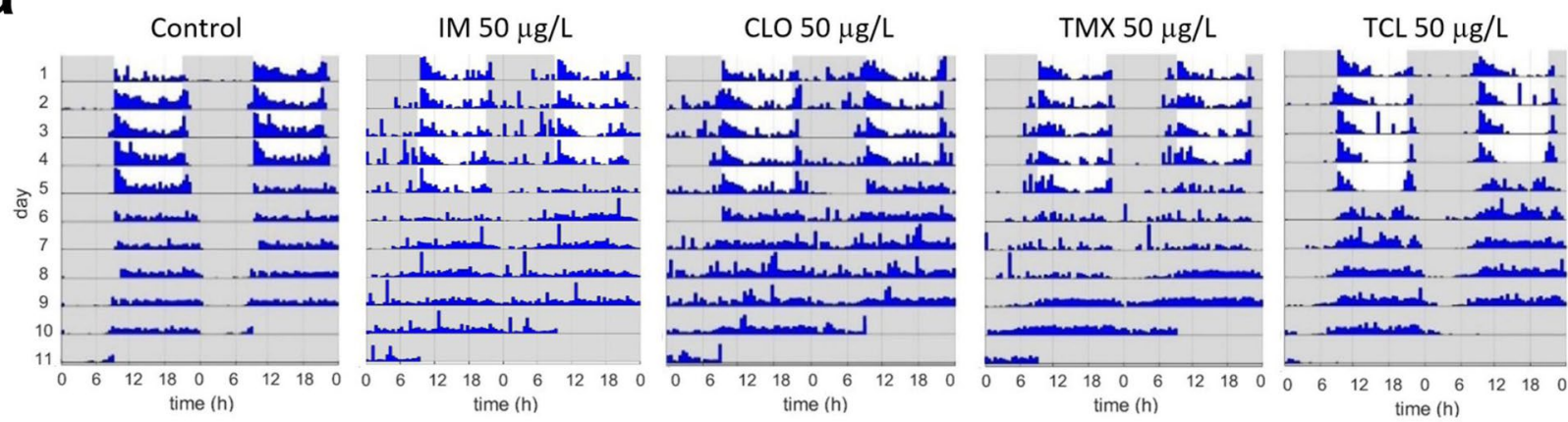

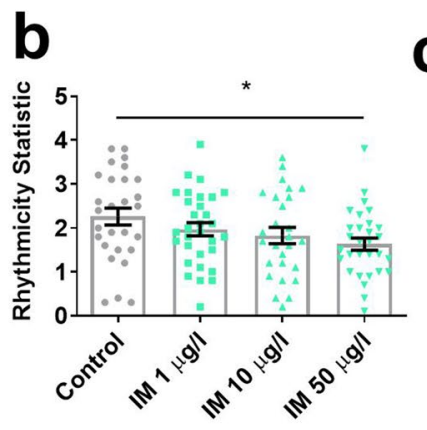

C

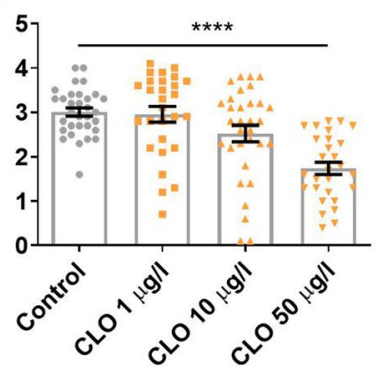

g
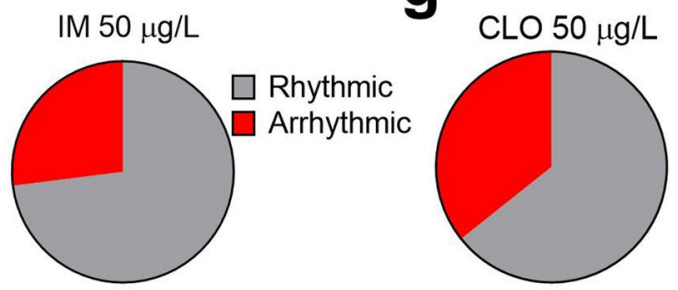

d

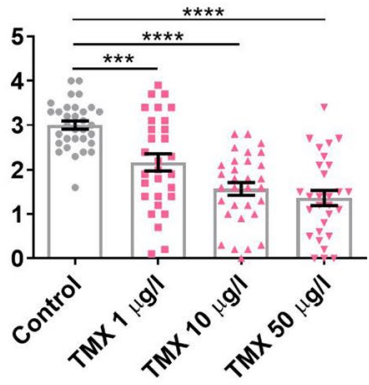

h

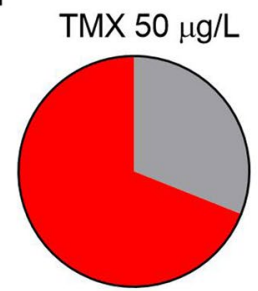

e

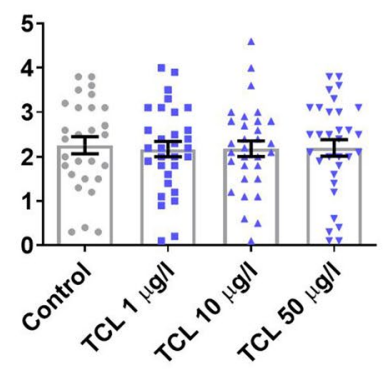

i

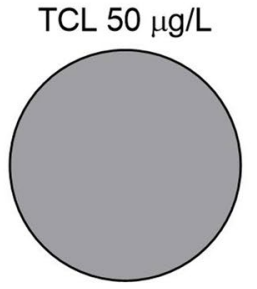

Figure 2. Field relevant concentrations of neonicotinoids reduced behavioural rhythmicity. (a) Representative actograms of the activity of single flies for control or $50 \mu \mathrm{g} / \mathrm{L}$ imidacloprid, clothianidin, thiamethoxam or thiacloprid. Blue bars indicate activity, grey background indicates lights off, white background lights on. Mean rhythmicity for flies exposed to 1,10 or $50 \mu \mathrm{g} / \mathrm{L}(\mathbf{b}) \operatorname{IM}\left(F_{3,112}=2.5, p=0.060\right)$, (c) CLO $\left(F_{3,116}=14.2, p<0.001\right)$, (d) $\operatorname{TMX}\left(F_{3,118}=23.7, p<0.001\right)$ and (e) TCL $\left(F_{3,118}=0.05, p=0.987\right)$. Each data point represents a single fly, $\mathrm{n}=28-32$ flies per treatment. Pie charts show the increase in the proportion of the population who were arrhythmic (rhythmicity statistic (RS) $\leq 1.5$ ) for $50 \mu \mathrm{g} / \mathrm{L}$ : (f) IM, (g) CLO, (h) TMX and (i) TCL, compared to controls.

and imidacloprid had a similar effect (Fig. 3e,g,i,k) but only for night-time sleep (Fig. 3a,c, Supplementary Fig. S8). Thiacloprid caused an increase in the number of night-time sleep episodes (Fig. 3h) and unlike the other neonicotinoids, caused a loss in daytime sleep (Fig. 3d, Supplementary Fig. 7) at every concentration tested, due to a reduction in daytime sleep episode length (Fig. 3l). This is likely due to the increase in daytime sleep latency observed in thiacloprid treated flies (Supplementary Fig. S9).

In order to localise the effects of neonicotinoids on sleep and circadian behaviour we knocked down Dal or $\mathrm{D} \beta 2$ in tim-expressing cells throughout the neuronal clock network (Fig. 4). This resulted in reduced behavioural rhythmicity (Fig. 4b,f) and shorter night-time sleep episodes (Fig. 4g,h) in $D \beta 2$ knockdown flies, and increased the number of sleep episodes seen in both $D \alpha 1$ and $D \beta 2$ knockdown flies (Fig. $4 \mathrm{~d}, \mathrm{~g}$ ). This again showed that loss of these subunits caused similar behavioural disruption as neonicotinoid exposure, suggesting a functional $\mathrm{nAChR}$ containing these subunits mediates the in vivo effects of these insecticides. In order to test this was indeed the case, RNAi flies were exposed to $50 \mu \mathrm{g} / \mathrm{L}$ of imidacloprid or clothianidin, a concentration sufficient to reduce rhythmicity in control flies. On flies that already had their D $\alpha 1$ or D 32 blocked genetically by expression of subunit specific RNAi expression throughout the tim-expressing clock cells, we found this caused no further loss of rhythmicity (Fig. 4i,j), confirming that neonicotinoid's in vivo effects were mediated through a receptor containing one or both of subunits in the clock.

Neonicotinoids remove day/night differences in synaptic remodelling and PDF accumulation in the $s$-LNvs dorsal terminals. To further characterise the mechanism by which neonicotinoids disrupt circadian rhythms day/night differences in synaptic remodelling and PDF cycling of the sLNv dorsal terminals were investigated. As previously reported ${ }^{45}$, in control flies the terminals were more branched and had higher 
a
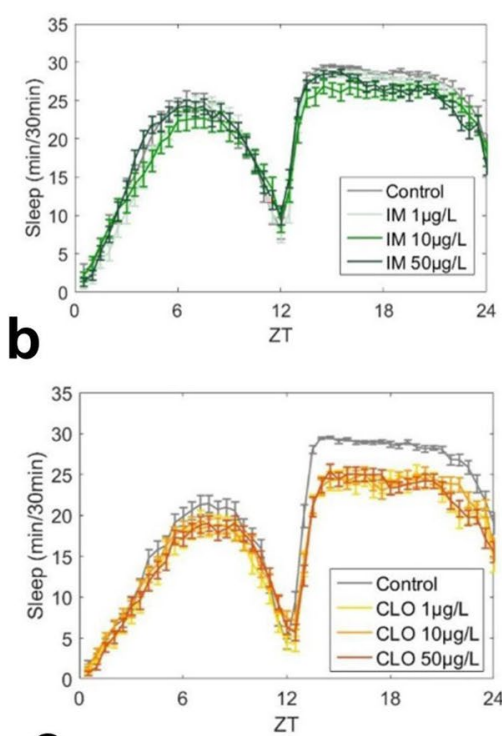

C

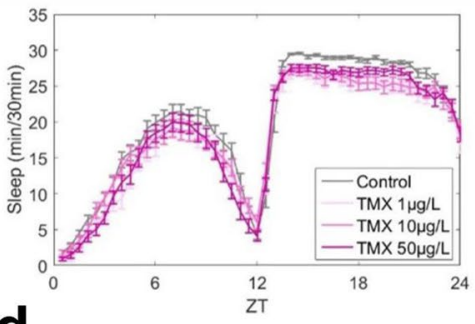

d

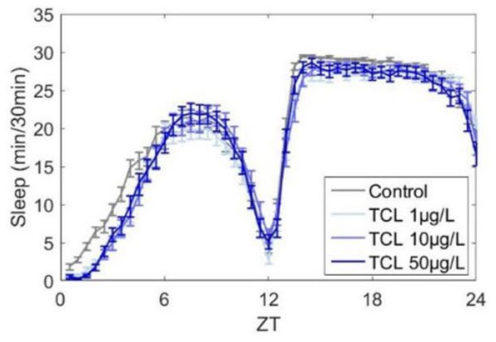

e
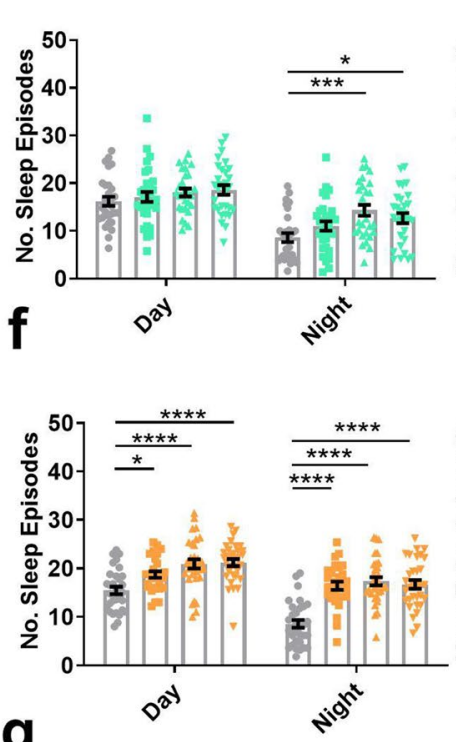

g
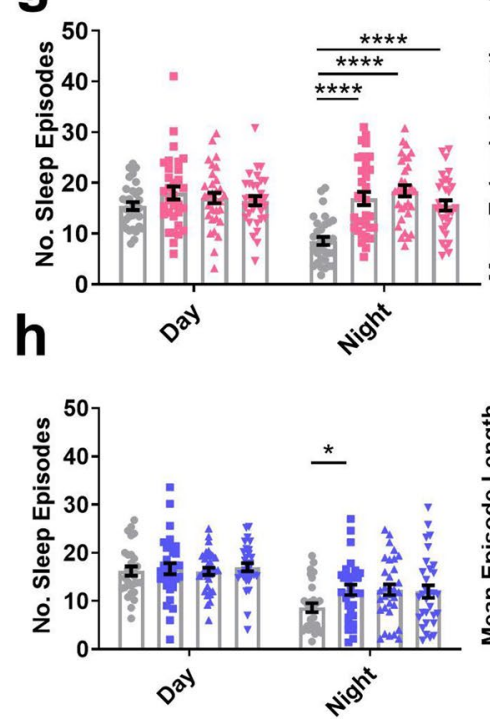

i

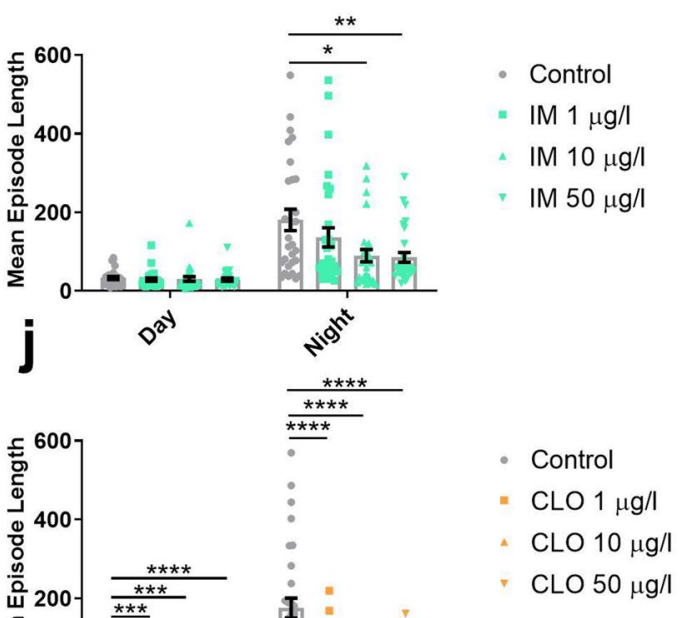

Figure 3. Field relevant concentrations of neonicotinoids disrupted sleep behaviour. Sleep plots showing the total sleep achieved per $30 \mathrm{~min}$ bin over the $24 \mathrm{~h}$ period (zeitgeber time (ZT)) for flies exposed to 1, 10 or $50 \mu \mathrm{g} / \mathrm{L}$ of (a) imidacloprid, (b) clothianidin, (c) thiamethoxam or (d) thiacloprid. The number of (no.) of sleep episodes initiated in (e) IM, day $\left(F_{3,114}=1.2, p=0.320\right)$ and night $\left(F_{3,114}=5.5, p=0.001\right)$, (f) CLO, day $\left(F_{3,120}=11.5, p<0.001\right)$ and night $\left(F_{3,120}=25.0, p<0.001\right)$, $(\mathrm{g}) \mathrm{TMX}$, day $\left(F_{3,124}=1.1, p=0.344\right)$ and night $\left(F_{3,124}=17.0, p<0.001\right)$ or $(\mathbf{h}) \mathrm{TCL}$, day $\left(F_{3,120}=0.2, p=0.872\right)$ and night $\left(F_{3,120}=3.0, p=0.034\right)$. Mean length (in minutes) of sleep episodes initiated in (i) IM, day $\left(F_{3,114}=0.2, p=0.889\right)$ and night $\left(F_{3,114}=4.5, p=0.005\right)$, (j) CLO, day $\left(F_{3,120}=9.9, p<0.001\right)$ and night $\left(F_{3,120}=21.8, p<0.001\right),(\mathbf{k}) \mathrm{TMX}$, day $\left(F_{3,124}=2.5, p=0.061\right)$ and night $\left(F_{3,124}=15.7, p<0.001\right)$ or $(\mathbf{l}) \mathrm{TCL}$, day $\left(F_{3,120}=5.2, p=0.002\right)$ and night $\left(F_{3,120}=2.0, p=0.121\right)$. Each data point represents a single fly, $n=28-32$ flies per treatment. CLO and TMX were run together with one set of controls, whilst IM and TCL were run together with another control group.

accumulation of PDF in the day than at night (Fig. 5a-c). In contrast, flies exposed to $50 \mu \mathrm{g} / \mathrm{L}$ imidacloprid or clothianidin showed no difference between day and night synaptic terminal branching or PDF accumulation (Fig. 5a-c). Likewise, in flies with knockdown of either D 11 or D 32 nAChRs in the PDF neurons, branching and PDF accumulation again showed no difference between day and night (Fig. 6a-c). Therefore the pharmacological and genetic manipulation of LNv nAChRs both similarly block the circadian output of these PDF expressing pacemaker neurons resulting in compromised circadian behaviour observed. 
a

\section{tim>Da1 RNAi}

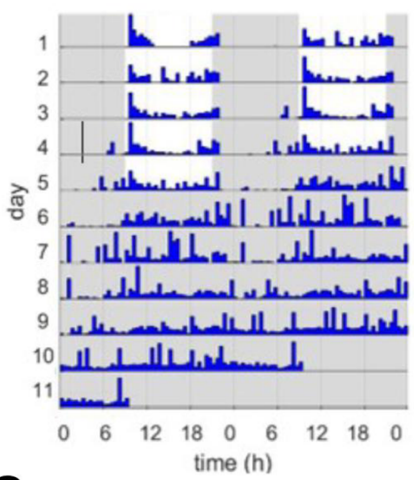

C
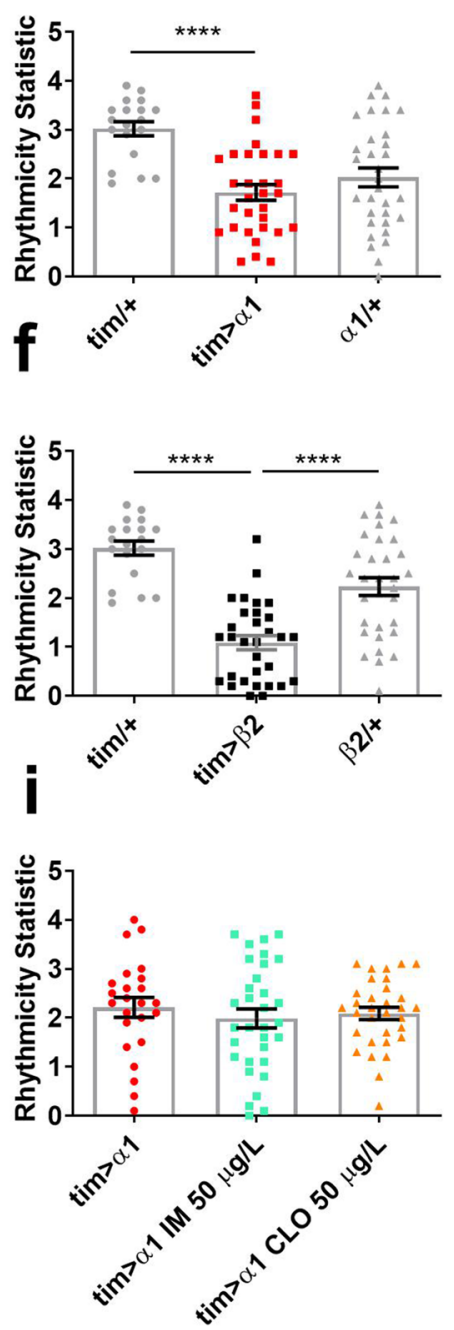

b

\section{tim>Dß2 RNAi}

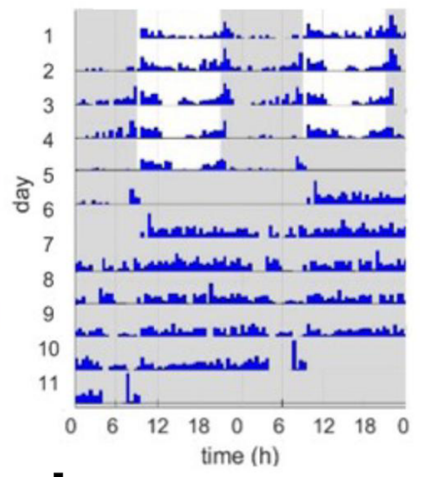

d
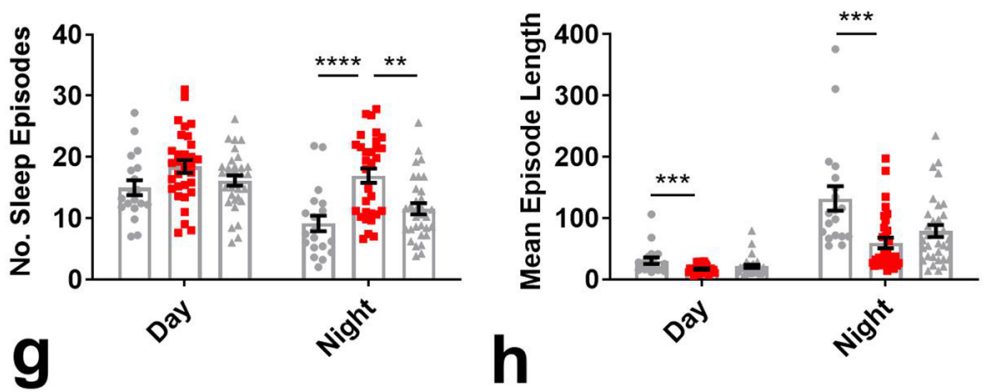

- tim/+

- tim>a1 RNAi

a1 RNAi/+
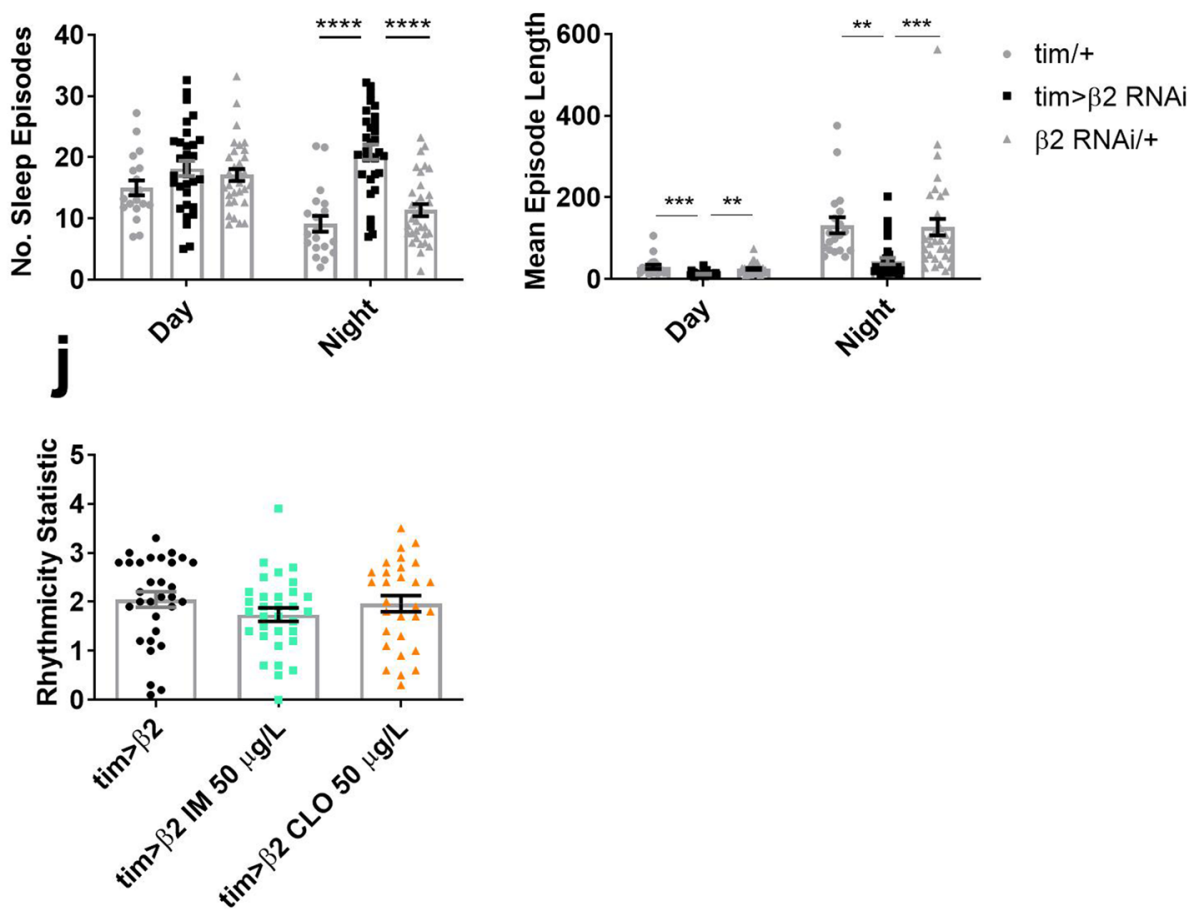

Figure 4. Knockdown of D $\alpha 1$ or $\mathrm{D} \beta 2$ in the clock bearing cells disrupts circadian rhythmicity and sleep with no further effect by addition of neonicotinoids. Representative actograms for (a) Dal knock down (timGal4 > uas- $n A C h R-D \alpha 1)$ and (b) D $\beta 2$ knockdown (tim-Gal4> uas-nAChR-D $\beta 2$. Effects of knocking down $D \alpha 1$ in clock bearing cells on (c) rhythmicity (RS) $\left(F_{2,79}=11.8, p<0.001\right)$, (d) number (no.) of sleep episodes in day $\left(F_{2,79}=2.9, p=0.063\right)$ and night $\left(F_{2,79}=12.3, p<0.001\right)$ and $(\mathbf{e})$ mean episode length in day $\left(F_{2,79}=5.1, p=0.008\right)$ and night $\left(F_{2,79}=8.3, p=0.001\right)$. Effects of knocking down $D \beta 2$ in clock bearing cells on (f) rhythmicity $\left(F_{2,79}=31.5, p<0.001\right)$, (g) no. of sleep episodes in day $\left(F_{2,79}=1.6, p=0.211\right)$ and night $\left(F_{2,79}=28.2, p<0.001\right)$ and (h) mean episode length in day $\left(F_{2,79}=11.2, p<0.001\right)$ and night $\left(F_{2,79}=9.4, p<0.001\right)$. Each data point represents a single fly, $n=19-32$ flies per treatment. There was no additive effect of $50 \mu \mathrm{g} / \mathrm{L}$ of IM and CLO on (i) tim $>\alpha 1$ $\left(F_{2,85}=0.4, p=0.677\right)$ and $(\mathbf{j})$ tim $>\beta 2\left(F_{2,89}=1.1, p=0.336\right)$. Each data point represents a single fly, $\mathrm{n}=24-32$ flies per treatment. 
a
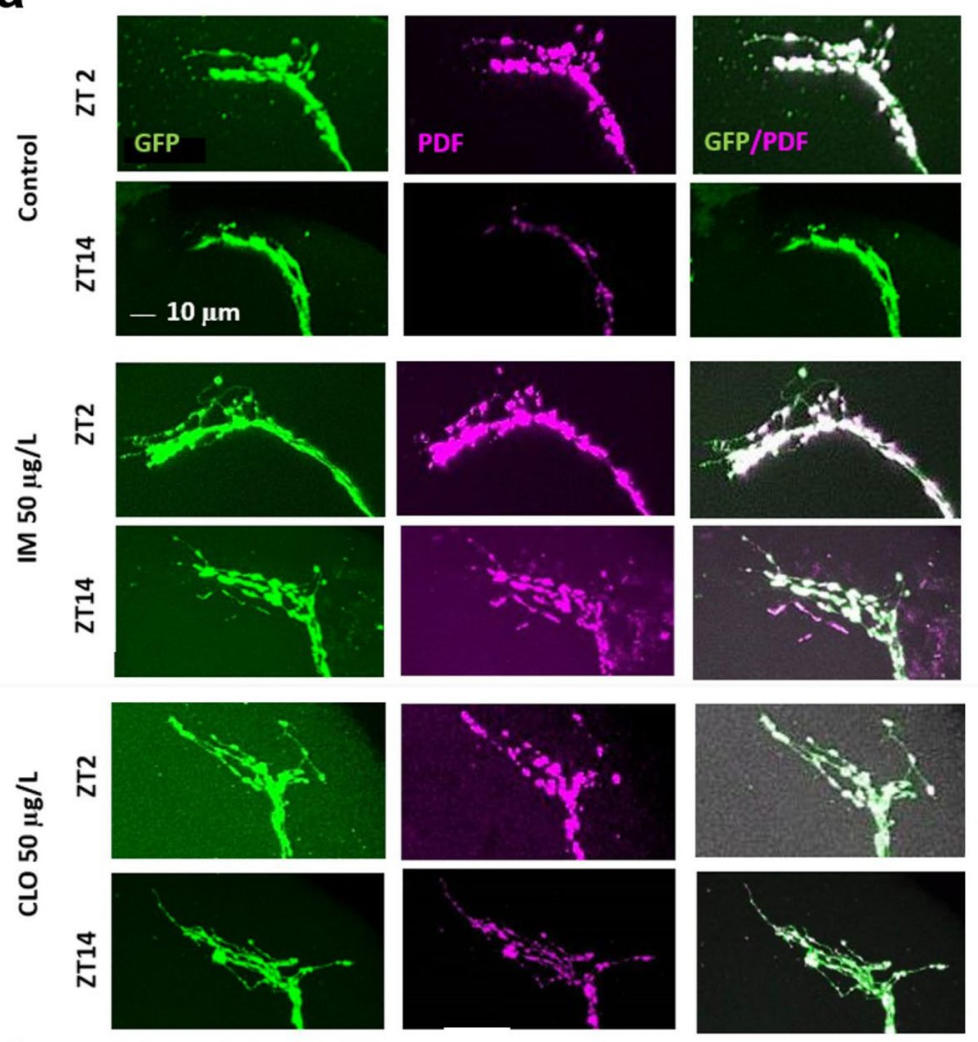

b

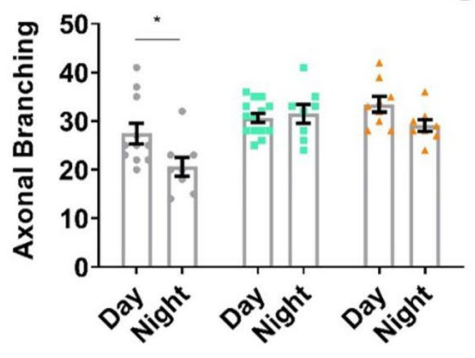

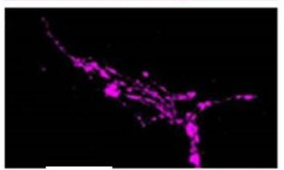

C

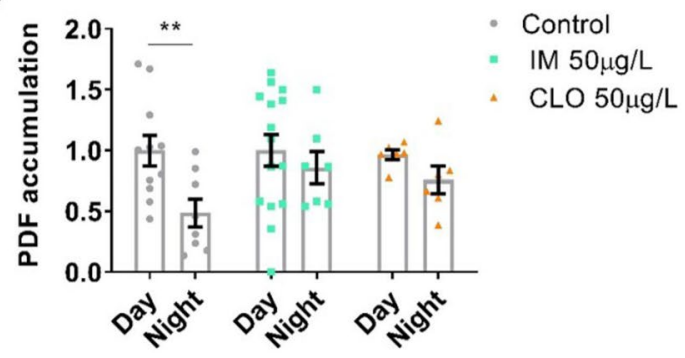

Figure 5. Field relevant concentrations of neonicotinoids disrupt the day/night remodelling and PDF cycling in the s-LNv clock neuron dorsal terminals. (a) Representative confocal images of the s-LNv dorsal terminals for control and treated (50 $\mu \mathrm{g} / \mathrm{L} \mathrm{IM}$ or CLO) flies in the day (ZT2 i.e. 11am) and night (ZT14 i.e. $11 \mathrm{pm})$. (b) s-LNv dorsal terminal branching complexity is greater in the day than at night for control flies $\left(t_{17}=2.3, p=0.036\right)$. The day/night differences in complexity is removed in flies exposed to $50 \mu \mathrm{g} / \mathrm{L}$ of IM $\left(t_{14}=2.1, p=0.055\right)$ or CLO $\left(t_{15}=2.1, p=0.052\right)$. (c) Accumulation of PDF in dorsal terminals is greater in the day than at night in control flies $\left(t_{17}=2.9, p=0.010\right)$, treatment with $50 \mu \mathrm{g} / \mathrm{L} \mathrm{IM}\left(t_{13}=1.0, p=0.332\right)$ or CLO $\left(t_{14}=2.1, p=0.054\right)$ removed this day/night difference in PDF levels. Each data point represents a single brain, $n=6-15$ brains.

\section{Discussion}

We showed that field relevant concentrations of all neonicotinoids tested had significant lethal effects on Drosophila, including decreased viability and shortened lifespan. In contrast the behavioural or sub-lethal effects on flies differed between the neonicotinoids with the nitro group-containing imidacloprid, clothianidin and thiamethoxam disrupting memory, locomotion, sleep and circadian behaviour, in contrast the cyano-group containing thiacloprid only caused fragmentation and reduction in sleep leaving the other behaviours intact. Therefore, thiacloprid appears to be less disruptive to behaviour than the other neonicotinoids tested. However its effects on sleep revealed significant sub-lethal effects even at the lowest level (1 ppb) reported to have sublethal effects by any neonicotinoid. In addition it decreased viability and caused early death, providing further evidence in support of the EU's extension of the neonicotinoid ban to thiacloprid. Clothianidin and thiamethoxam showed the greatest effects, which is consistent with them being full agonists at nAChRs and many other studies in pollinators finding them to be more toxic and potent than the partial agonist imidacloprid ${ }^{9,52,59}$. The sub-lethal effects we report in Drosophila are also consistent with those reported for pollinators that show the nitro-containing neonicotinoids (clothianidin, thiamethoxam and imidacloprid) are generally more toxic than the cyano-group containing neonicotinoids (thiacloprid) $)^{1,9-11,52}$. 


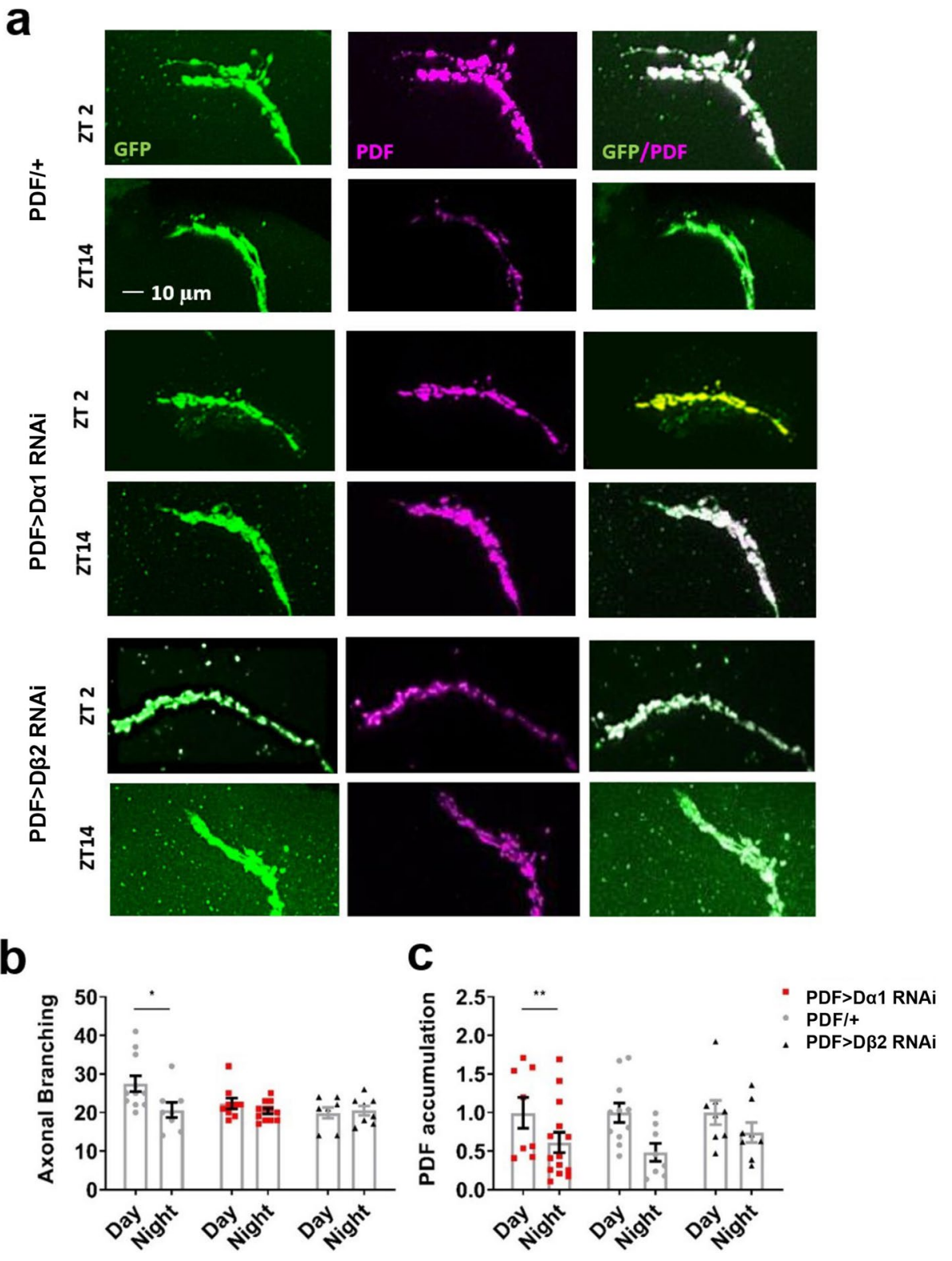

Figure 6. Knockdown of Da1 or D 32 disrupted the day/night remodelling and PDF cycling of the s-LNv dorsal terminals. (a) Representative confocal images of the s-LNv dorsal terminals of control flies $(P D F-G a l 4 /+)$ and flies with Da1 (PDF-Gal4> uas-nAChR-D $\alpha 1-R N A i)$ and D $\beta 2$ (PDF-Gal4> uas-nAChR-D $\beta 2-R N A i)$ knocked down in LNv clock neurons taken in the day (ZT2) and night (ZT14). (b) The s-LNv dorsal terminals of control flies showed greater branching complexity in the day than at night $\left(t_{17}=2.3, p=0.036\right)$, this day/night difference in terminal complexity was removed in $P D F>D \alpha 1-R N A i\left(t_{19}=1.4, p=0.183\right)$ and $P D F>D \beta 2-R N A i\left(t_{13}=-0.7\right.$, $p=0.515$ ) flies. (c) PDF accumulation in the s-LNv dorsal terminals was greater in the day than at night for control flies $\left(t_{17}=2.9, p=0.010\right)$, but not in $P D F>D \alpha 1-R N A i$ flies $\left(t_{19}=1.8, p=0.089\right)$ and $P D F>D \beta 2-R N A i$ flies $\left(t_{14}=1.3, p=0.218\right)$. Each data point represents a single brain, $\mathrm{n}=6-15$ brains.

The effects of field-relevant concentrations of neonicotinoids on memory in Drosophila, reiterates the conserved toxicity and sub-lethal effects of the insecticides on non-target insects. That lack of effect of neonicotinoids on fly olfaction and to shock reactivity, confirmed that the neonicotinoids interfered with memory formation itself as opposed to detection of cues. This was also supported by the data showing that knockdown of the neonicotinoid susceptible D $\alpha 1$ or D $\beta 2$ subunits in just the mushroom body was sufficient to cause similar memory deficits as observed in the neonicotinoid exposed flies. The Kenyon cells of the mushroom body are cholinergic ${ }^{59}$, contain $\mathrm{nAChRs}{ }^{60}$ and the postsynaptic mushroom body output neuron also signal via nAChRs ${ }^{24}$. Therefore, neonicotinoids can act at multiple nAChR synapses in the mushroom body circuit, disrupting the plasticityrelevant signals for memory formation. Furthermore, in bees, field relevant concentrations of neonicotinoids disrupted mushroom body-mediated olfactory memory, electrically inactivated Kenyon cells ${ }^{22}$, decreased their synaptic density ${ }^{26}$ and reduced antennal lobe $\mathrm{Ca}^{2+}$ responses upstream of the mushroom body ${ }^{27}$. Additionally, 
neonicotinoids have been shown to interrupt mushroom body development ${ }^{27}$, providing a further route for memory disruption. Our results extend existing data from pollinators showing neonicotinoid disrupt memory by showing for the first time that the disruption is mediated by nAChRs containing Da1 or D $\beta 2$ subunits in the mushroom body. Furthermore, given that neonicotinoids appear to disrupt circadian rhythmicity, and given that there is circadian regulation of memory formation with time of day influencing performance, neonicotinoidinduced disruption to the clock may contribute to the memory deficits ${ }^{61}$. The time-of-day differences in mushroom body memory are thought to result from day/night differences in PDF and PDFR signalling ${ }^{30}$, therefore the loss sLNv dorsal terminal PDF rhythmic signalling of PDF caused by the neonicotinoids may provide another route to the insecticide induced reductions in memory we report.

Similarly, the sleep and circadian effects caused by neonicotinoid exposure appear to be due to the neonicotinoids acting directly upon the clock. Knockdown of D $32 \mathrm{nAChR}$ subunit in the clock neurons resulted in a similar disruption of rhythmicity as exposure to field-relevant concentrations of neonicotinoids, whilst knockdown of Da1 or $\mathrm{D} \beta 2$ caused changes to sleep behaviour reflecting those seen in neonicotinoid exposed flies. This suggests that D $\alpha 1$ and $\mathrm{D} \beta 2$ mediate the effects of neonicotinoids on clock neurons resulting in the disruption of circadian rhythms and sleep. Consistent with this, exposure of $\mathrm{D} \beta 2$ knockdown flies to imidacloprid or clothianidin caused no further effect on circadian rhythmicity confirming that $\mathrm{D} \beta 2$ in clock neurons mediates the in vivo effect of neonicotinoids on circadian rhythms.

This is consistent with studies that have shown the LNvs usually receive excitatory ACh inputs from the lightsensing organs $s^{34,35}$ and express nAChRs ${ }^{39,42}$, further field relevant concentrations of neonicotinoids have been shown to directly increase LNv neural activity ${ }^{23}$. The electrical state of these pacemaker neurons influences their circadian output including the circadian remodelling of the s-LNv dorsal terminals and circadian abundance of $\mathrm{PDF}^{43,62}$, with the release of this neuropeptide being necessary for behavioural rhythmicity ${ }^{40}$. We found that neonicotinoid exposure caused a loss of PDF cycling and terminal morphological plasticity, with the terminals remaining in a branched, day state continuously, suggesting that the agonist effect of neonicotinoids disrupt normal day-night differences in the electrical state of these neurons that is required for circadian rhythms $\mathrm{s}^{35,36,43,44,58}$. Indeed, nAChR synaptic signalling is involved in the rhythmic firing of action potentials in clock neurons of Drosophila and other insects ${ }^{36,42,63}$. Furthermore field relevant concentrations of imidacloprid have also shown to cause degeneration of retinae and reduced visual acuity, providing a further possible route for neonicotinoids to disrupt light input into the clock, and hence the structural and behavioural changes observed here ${ }^{47}$.

Knock-down of the neonicotinoid susceptible nAChR subunits D $\alpha 1$ or D $\beta 2$ also removed day-night differences in the terminals reminiscent of the changes seen in flies with electrically silenced LNvs ${ }^{62}$. The agonist action of neonicotinoids on nAChRs on the LNvs we and others have demonstrated ${ }^{23}$ also explains the sleep disruption we reported as the l-LNv electrical state is vital to their role as arousal neurons, with l-LNv hyperexcitation leading to sleep defects such as loss of night-time sleep and shorter sleep episodes ${ }^{37,64}$, effects recapitulated in the neonicotinoid exposed flies.

The high degree of structural and functional conservation of nAChRs between flies and bees ${ }^{9,50,65}$, and the conserved lethal and sub-lethal effects of reduced viability, longevity, locomotion, memory, circadian rhythmicity and sleep we show in flies and as reported in bees suggests that the modes of action identified here may also occur in bees ${ }^{1,21,23,33,53-56}$. Previous work has shown that sub-lethal effects observed in the lab translate to the field, for example neonicotinoid reduced foraging motivation is observed in bumblebees in the lab as well as in free flying colonies in the field ${ }^{20,66}$. Reduced behavioural rhythmicity, shown in honeybees ${ }^{23,33}$, bumblebees ${ }^{21}$ and here in fruit flies, is likely to reduce the amount of activity carried out in the daytime, reducing pollination and foraging opportunities ${ }^{67}$. The reduction in total sleep and fragmentation of sleep will reduce the quantity of deep sleep achieved, as deep sleep occurs later into the sleep episode ${ }^{68}$. It is known that deep sleep is particularly important for memory consolidation ${ }^{32}$, therefore this is likely to further compound the effects of neonicotinoids on memory, which will again impact foraging efficiency.

In summary, $\mathrm{nAChR}$ subunits D 1 and $\mathrm{D} \beta 2$ expression in the mushroom body appears to mediate the effect of field-relevant concentrations of nitro-containing imidacloprid, clothianidin and thiamethoxam on memory, while expression of these subunits in the clock mediates the effect of neonicotinoids on circadian rhythmicity and sleep. The more recently banned cyano-containing neonicotinoid thiacloprid was less toxic, only disrupting sleep, which seems to be the most sensitive behavioural metric of the sub-lethal effects of neonicotinoids. In addition, all four of the neonicotinoids tested decreased both viability and shortened lifespan, therefore supporting the current EU ban. This work illustrates the utility of neonicotinoids as a pharmacological tool for exploration of $\mathrm{nAChR}$ function, as well as the use of Drosophila in revealing the mechanism of action of neonicotinoids and elucidating the sub-lethal and lethal effects of these insecticides, highlighting their potential impact on insects in the field.

\section{Materials and methods}

Fly husbandry and genotypes. The following fly stocks were used: wild-type strains iso31 (Gift from Dr Ralph Stanewsky, University of Münster, Germany) for climbing, circadian and sleep assays and CSw- (gift from Dr Scott Waddell, University of Oxford, UK) for all other experiments, Pdf-Gal4 (Bloomington Drosophila stock center number (BDSC): 6900), elav-Gal4 (BDSC: 8760), tim $^{28}$-Gal4 (BDSC: 80976, gift were from Dr Ralf Stanewsky, University of Münster, Germany), OK107-Gal4 (BDSC: 854), uas-mcd8::GFP (BDSC: 5137), uas$n A C h R-D \alpha 1-R N A i$ (BDSC: 28688), and UAS- $n A C h R-D \beta 2-R N A i$ (BDSC: 28038). For all experiments, flies were collected shortly after eclosion and used within 5 days. For climbing, circadian, sleep, longevity, immunohistochemistry and offspring survival assays females were used, for learning and memory mixed sex groups were used.

Flies were bred, maintained and tested on standard polenta-based food mixture at $25^{\circ} \mathrm{C}, 55-65 \%$ humidity under $12 \mathrm{~h}$ light: $12 \mathrm{~h}$ dark (LD) conditions. Food was made up in $5 \mathrm{~L}$ quantities and contained: $400 \mathrm{~g}$ polenta, 
$35 \mathrm{~g}$ granulated agar, $90 \mathrm{~g}$ active dried yeast, $50 \mathrm{~g}$ soya flour, $400 \mathrm{~mL}$ malt extract and $200 \mathrm{~mL}$ molasses, with $40 \mathrm{~mL}$ of propionic acid (Sigma-Aldrich, \#94425) and $100 \mathrm{~mL}$ of nipagin (Sigma-Aldrich, \#H5501) added once cool. Neonicotinoids were added to food before it set from a frozen and aliquoted stock solution of 100,000 $\mu \mathrm{g} / \mathrm{L}$ $\mathrm{ddH}_{2} \mathrm{O}$. The neonicotinoids were analytical standard (PESTANAL Sigma-Aldrich): imidacloprid, clothianidin, thiamethoxam, and thiacloprid.

Aversive olfactory conditioning. $1 \mathrm{~h}$ memory was tested using aversive olfactory conditioning ${ }^{57}$. Groups of 30-50 one-five days old mixed sex flies were reared on control or neonicotinoid containing food. Flies were exposed consecutively to one of two odours, either 4-methylcyclohexanol (Sigma) or 3-octanol (Sigma) diluted 1:500 and 1:250 respectively, paired with $1.25 \mathrm{~s}$ pulses of $70 \mathrm{~V}$ electric shock, with $3.75 \mathrm{~s}$ pauses between shocks. Flies were then returned to food vials for $1 \mathrm{~h}$ before memory was tested. For testing, flies were loaded into the choice point of the T-maze and, after a $90 \mathrm{~s}$ acclimatisation period, were given the choice of two tubes, one containing each of the test odours. Flies were given $2 \mathrm{~min}$ and then the proportion in each arm was counted. A separate group of flies were then trained and tested with the reciprocal odour. The performance index score represents the proportion of flies who correctly avoided the arm containing the odour which had been delivered with shock during training, as shown below.

$\mathrm{PI}=$ (number of correct flies - number of incorrect flies)/total number of flies.

The PI for flies shocked with each odour separately were averaged to give an $n=1$.

Circadian rhythms. Behavioural rhythmicity data was collected using Drosophila Activity Monitors (DAM2, TriKinetics Inc ${ }^{69}$. Virgin females were placed in individual tubes in the DAM, with control or neonicotinoid containing food, 32 flies per treatment, for 5 days in LD followed by 5 days constant darkness (DD). Flies who died before the end of day ten were not included in analysis. DAM tubes were intersected with an infrared beam, with each beam cross counted as an activity bout. To quantify circadian rhythmicity, the data was summed into $30 \mathrm{~min}$ bins. From this an actogram was created and rhythmicity statistic and period length were calculated for each individual fly for the DD portion using Flytoolbox ${ }^{70}$ in MATLAB (MATLAB and Statistics Toolbox Release 2012b, The MathWorks). The proportion of flies in each treatment group whose Rhythmicity Statistic was below 1.5, generally considered to denote arrhythmicity ${ }^{70}$, was calculated. This was then displayed in a pie chart for each group, after being normalised by removing the proportion of controls who were arrhythmic for each run. The rhythmicity of each fly during the LD section of the run was also calculated.

Sleep. For analysis of sleep behaviour, flies were loaded into the DAM as described above. Sleep measures were extracted from activity data from 5 days of LD, summed into $1 \mathrm{~min}$ and $30 \mathrm{~min}$ bins. Sleep was defined as bouts of inactivity lasting more than $5 \mathrm{~min}$, as is convention ${ }^{70,71}$. The mean total sleep, mean sleep episode length, mean number of sleep episodes per day and night and mean sleep latency were calculated for each individual using the Sleep and Circadian Analysis MATLAB Program (SCAMP) in MATLAB ${ }^{72}$. For each treatment, a mean sleep profile was also plotted showing mean sleep quantity per $30 \mathrm{~min}$ bin over the $24 \mathrm{~h}$ period.

Imaging the arborisation and PDF cycling of s-LNv dorsal terminals. Immunohistochemistry was adapted from the method described in Fernández et al. ${ }^{45}$. Virgin females were collected and placed in vials. After 5 days LD, on control or neonicotinoid food, flies were anesthetised using $\mathrm{CO}_{2}$ at either $2 \mathrm{~h}$ after lights on (ZT2) or $2 \mathrm{~h}$ after lights off (ZT14) and decapitated. Heads were fixed in phosphate-buffered solution (PBS) with $4 \%$ paraformaldehyde (Thermo Fisher Scientific) containing 0.008\% Triton X-100 (Sigma-Aldrich) for 45 min at room temperature. Heads were washed quickly twice in PBS with $0.5 \%$ Triton X-100 (PBT 0.5\%), followed by three 20 min washes in PBT $0.5 \%$ and dissection in PBT $0.1 \%$. Brains were blocked in 5\% Normal Goat Serum (NGS, Thermo Fisher) for $1 \mathrm{~h}$, then incubated for $36 \mathrm{~h}$ at $4{ }^{\circ} \mathrm{C}$ with mouse monoclonal anti-PDF (Developmental Studies Hybridoma Bank, \#PDF-C7) and rabbit polyclonal anti-GFP (Life Technologies \# A11122) in NGS at concentrations of 1:200 and 1:1000 respectively.

Brains were washed again as before and then incubated for $3 \mathrm{~h}$ at room temperature followed by $24 \mathrm{~h}$ at $4{ }^{\circ} \mathrm{C}$ with Alexa Fluor Plus 488 Goat anti-mouse (Life Technologies \# A32723) and Alexa Fluor Plus 555 goat anti-rabbit (Life Technologies \# A32732) in NGS at concentrations of 1:1000 and 1:100 respectively. Brains were washed once more and then mounted onto glass slides using spacers (SecureSeal, Grace Bio-Labs \#654002), covered with VectaShield hard set medium (Vector Laboratories) and secured with CoverGrip (Biotium \#23005).

Imaging was carried out on a Leica SPE confocal laser scanning microscope with the green channel imaged at $480-551 \mathrm{~nm}$ and the red at 571-650 $\mathrm{nm}$. Z stacks were captured of the s-LNv dorsal terminals using a $64 \times$ oil immersion objective, with a step size of $2 \mu \mathrm{m}$. Maximal projection stacks were created and analysed using FIJI $(\text { Image J })^{73}$. The arborisation of each s-LNv terminal was calculated using an adaptation of the Scholl analysis ${ }^{45}$. Six concentric rings $10 \mu \mathrm{m}$ apart were drawn, centred at the first branching point and the number of branches touching each ring was summed. Both hemispheres were measured. Mean scores for ZT2 and ZT14 in each group were compared using Pearson's $t$-test using SPSS Statistics 24 (IBM Corporation).

For PDF staining intensity, the image was cut at the first branching point to create an image containing only the terminals and not the cell axon and the image was analysed in FIJI (ImageJ). The image was transformed into 8-bit and the threshold adjusted to create a black and white image. The de-speckle filter was used to reduce noise and watershed segmentation carried out to separate the different PDF compartments. This image was then used as a template for calculating the PDF staining in the original maximal projection image, allowing the PDF staining intensity to be calculated for each of these compartments and the mean taken. The mean for both hemispheres of the brain was calculated and reported, and the means for ZT2 and ZT14 were compared as for axonal branching. 
Statistical analysis. Normality of the data was checked using a Shapiro-Wilk test. Data were also checked for homogeneity of variance using Levene's test for equality of variances. Unless otherwise stated, means were then compared using a one-way ANOVA with post hoc pairwise comparisons being carried out using Tukey's multiple comparisons test. Where data were not normally distributed, a Kruskal-Wallis one-way ANOVA was carried out with Dunnett's multiple comparison. Statistical analysis was done in SPSS Statistics 24 (IBM Corporation). Graphs were created in GraphPad (Prism version 8.0.0 for Windows, GraphPad Software).

The sleep data failed the assumptions of normal distribution and homogeneity of differences. Thus, permutation tests were conducted in R 3.4.1. As the resulting $p$ values closely matched those produced by analysing the same data using a one-way ANOVA as above, and because ANOVA is relatively robust to deviations from normality when sample sizes are large, the results of the one-way ANOVA were displayed.

\section{Data availability}

The datasets generated during and/or analysed during the current study are available in the Mendeley Data repository, under 'Neonicotinoids disrupt memory, circadian behaviour and sleep, Tasman et al., https://doi. org/10.17632/d28dk6znm6.1

Received: 29 June 2020; Accepted: 28 December 2020

Published online: 21 January 2021

\section{References}

1. Wood, T. J. \& Goulson, D. The environmental risks of neonicotinoid pesticides: a review of the evidence post 2013. Environ. Sci. Pollut. Res. Int. 24, 17285-17325. https://doi.org/10.1007/s11356-017-9240-x (2017).

2. Wagner, D. L. Insect declines in the anthropocene. Annu. Rev. Entomol. 65, 457-480. https://doi.org/10.1146/annurev-ento-01101 9-025151 (2020).

3. Klein, A.-M. et al. Importance of pollinators in changing landscapes for world crops. Proc. R. Soc. B Biol. Sci. 274, 303-313. https ://doi.org/10.1098/rspb.2006.3721 (2007).

4. Gallai, N., Salles, J.-M., Settele, J. \& Vaissière, B. E. Economic valuation of the vulnerability of world agriculture confronted with pollinator decline. Ecol. Econ. 68, 810-821. https://doi.org/10.1016/j.ecolecon.2008.06.014 (2009).

5. Hallmann, C. A. et al. More than 75 percent decline over 27 years in total flying insect biomass in protected areas. PLoS ONE 12, e0185809. https://doi.org/10.1371/journal.pone.0185809 (2017).

6. Goulson, D. The insect apocalypse, and why it matters. Curr. Biol. 29, R967-R971. https://doi.org/10.1016/j.cub.2019.06.069 (2019).

7. Casida, J. E. \& Durkin, K. A. Neuroactive insecticides: targets, selectivity, resistance, and secondary effects. Annu. Rev. Entomol. 58, 99-117. https://doi.org/10.1146/annurev-ento-120811-153645 (2013).

8. Popp, J., Pető, K. \& Nagy, J. Pesticide productivity and food security. A review. . Agron. Sustain. Dev. 33, 243-255. https://doi. org/10.1007/s13593-012-0105-x (2013).

9. Casida, J. E. Neonicotinoids and other insect nicotinic receptor competitive modulators: progress and prospects. Annu. Rev. Entomol. 63, 125-144. https://doi.org/10.1146/annurev-ento-020117-043042 (2018).

10. Matsuda, K., Ihara, M. \& Sattelle, D. B. Neonicotinoid insecticides: molecular targets, resistance, and toxicity. Annu. Rev. Pharmacol. Toxicol. 60, 241-255. https://doi.org/10.1146/annurev-pharmtox-010818-021747 (2020).

11. Goulson, D. REVIEW: an overview of the environmental risks posed by neonicotinoid insecticides. J. Appl. Ecol. 50, 977-987. https://doi.org/10.1111/1365-2664.12111 (2013).

12. Eng, M. L., Stutchbury, B. J. M. \& Morrissey, C. A. A neonicotinoid insecticide reduces fueling and delays migration in songbirds. Science 365, 1177. https://doi.org/10.1126/science.aaw9419 (2019).

13. Yamamuro, M. et al. Neonicotinoids disrupt aquatic food webs and decrease fishery yields. Science (New York, N.Y.) 366, 620. https ://doi.org/10.1126/science.aax3442 (2019).

14. Han, W., Tian, Y. \& Shen, X. Human exposure to neonicotinoid insecticides and the evaluation of their potential toxicity: an overview. Chemosphere 192, 59-65. https://doi.org/10.1016/j.chemosphere.2017.10.149 (2018).

15. Nauen, R., Ebbinghaus-Kintscher, U., Salgado, V. L. \& Kaussmann, M. Thiamethoxam is a neonicotinoid precursor converted to clothianidin in insects and plants. Pestic. Biochem. Physiol. 76, 55-69. https://doi.org/10.1016/S0048-3575(03)00065-8 (2003).

16. EFSA. Peer review of the pesticide risk assessment of the active substance thiacloprid. Eur. Food Saf. Auth. J. 17, e05595 https:// doi.org/10.2903/j.efsa.2019.5595 (2019).

17. Nicholls, E. et al. Monitoring neonicotinoid exposure for bees in rural and peri-urban areas of the U.K. during the transition from pre- to post-moratorium. Environ. Sci. Technol. 52, 9391-9402. https://doi.org/10.1021/acs.est.7b06573 (2018).

18. Wintermantel, D. et al. Neonicotinoid-induced mortality risk for bees foraging on oilseed rape nectar persists despite EU moratorium. Sci. Total Environ. 704, 135400. https://doi.org/10.1016/j.scitotenv.2019.135400 (2020).

19. Cressey, D. Fears for bees as UK lifts insecticide ban. Nature News. https://www.nature.com/news/fears-for-bees-as-uk-lifts-insec ticide-ban-1.18052 (2015).

20. Lamsa, J., Kuusela, E., Tuomi, J., Juntunen, S. \& Watts, P. C. Low dose of neonicotinoid insecticide reduces foraging motivation of bumblebees. Proc. R. Soc. B 285, 20180506 https://doi.org/10.1098/rspb.2018.0506 (2018).

21. Tasman, K., Rands, S. A. \& Hodge, J. J. The neonicotinoid insecticide imidacloprid disrupts bumblebee foraging rhythms and sleep. iScience 23, 101827 https://doi.org/10.2139/ssrn.3586989 (2020).

22. Palmer, M. J. et al. Cholinergic pesticides cause mushroom body neuronal inactivation in honeybees. Nat. Commun. 4, 1634. https ://doi.org/10.1038/ncomms2648 (2013).

23. Aso, Y. et al. Mushroom body output neurons encode valence and guide memory-based action selection in Drosophila. eLife 3, e04580. https://doi.org/10.7554/eLife.04580 (2014).

24. Barnstedt, O. et al. Memory-relevant mushroom body output synapses are cholinergic. Neuron 89, 1237-1247. https://doi. org/10.1016/j.neuron.2016.02.015 (2016).

25. Helfrich-Förster, C. Sleep in insects. Annu. Rev. Entomol. 63, 69-86. https://doi.org/10.1146/annurev-ento-020117-043201 (2018).

26. Peng, Y. C. \& Yang, E. C. Sublethal dosage of imidacloprid reduces the microglomerular density of honey bee mushroom bodies. Sci. Rep. 6, 19298. https://doi.org/10.1038/srep19298 (2016).

27. Smith, D. B. et al. Insecticide exposure during brood or early-adult development reduces brain growth and impairs adult learning in bumblebees. Proc. R. Soc. B 287, 20192442. https://doi.org/10.1098/rspb.2019.2442 (2020).

28. Andrione, M., Vallortigara, G., Antolini, R. \& Haase, A. Neonicotinoid-induced impairment of odour coding in the honeybee. Sci. Rep. 6, 38110. https://doi.org/10.1038/srep38110 (2016).

29. Chouhan, N. S., Wolf, R., Helfrich-Förster, C. \& Heisenberg, M. Flies remember the time of day. Curr. Biol. 25, 1619-1624. https ://doi.org/10.1016/j.cub.2015.04.032 (2015). 
30. Flyer-Adams, J. G. et al. Regulation of olfactory associative memory by the circadian clock output signal Pigment-dispersing factor (PDF). J. Neurosci. 40, 9066-9077https://doi.org/10.1523/JNEUROSCI.0782-20.2020 (2020).

31. Zwaka, H. et al. Context odor presentation during sleep enhances memory in honeybees. Curr. Biol. 25(21), 869-2874. https:// doi.org/10.1016/j.cub.2015.09.069 (2015).

32. Seugnet, L., Suzuki, Y., Donlea, J. M., Gottschalk, L. \& Shaw, P. J. Sleep deprivation during early-adult development results in long-lasting learning deficits in adult Drosophila. Sleep 34, 137-146. https://doi.org/10.1093/sleep/34.2.137 (2011).

33. Tackenberg, M. C. et al. Neonicotinoids disrupt circadian rhythms and sleep in honey bees. Sci. Rep. 10, 17929. https://doi. org/10.1038/s41598-020-72041-3 (2020).

34. Helfrich-Forster, C. et al. The extraretinal eyelet of Drosophila: development, ultrastructure, and putative circadian function. J. Neurosci. 22, 9255-9266. https://doi.org/10.1523/JNEUROSCI.22-21-09255.2002 (2002).

35. Muraro, N. I. \& Ceriani, M. F. Acetylcholine from visual circuits modulates the activity of arousal neurons in Drosophila. J. Neurosci. 35, 16315. https://doi.org/10.1523/JNEUROSCI.1571-15.2015 (2015).

36. McCarthy, E. V. et al. Synchronized bilateral synaptic inputs to Drosophila melanogaster neuropeptidergic rest/arousal neurons. J. Neurosci. 31, 8181-8193. https://doi.org/10.1523/jneurosci.2017-10.2011 (2011).

37. Parisky, K. M. et al. PDF cells are a GABA-responsive wake-promoting component of the Drosophila sleep circuit. Neuron 60, 672-682. https://doi.org/10.1016/j.neuron.2008.10.042 (2008).

38. Ly, S., Pack, A. I. \& Naidoo, N. The neurobiological basis of sleep: Insights from Drosophila. Neurosci. Biobehav. Rev. 87, 67-86. https://doi.org/10.1016/j.neubiorev.2018.01.015 (2018).

39. Wegener, C., Hamasaka, Y. \& Nassel, D. R. Acetylcholine increases intracellular $\mathrm{Ca}^{2+}$ via nicotinic receptors in cultured PDFcontaining clock neurons of Drosophila. J. Neurophysiol. 91, 912-923. https://doi.org/10.1152/jn.00678.2003 (2004).

40. Renn, S. C., Park, J. H., Rosbash, M., Hall, J. C. \& Taghert, P. H. A pdf neuropeptide gene mutation and ablation of PDF neurons each cause severe abnormalities of behavioral circadian rhythms in Drosophila. Cell 99, 791-802. https://doi.org/10.1016/s0092 -8674(00)81676-1 (1999).

41. Schlichting, M., Menegazzi, P., Rosbash, M. \& Helfrich-Förster, C. A distinct visual pathway mediates high-intensity light adaptation of the circadian clock in Drosophila. J. Neurosci. 39, 1621. https://doi.org/10.1523/JNEUROSCI.1497-18.2018 (2019).

42. Lelito, K. \& Shafer, O. Reciprocal cholinergic and GABAergic modulation of the small ventrolateral pacemaker neurons of Drosophila's circadian clock neuron network. J. Neurophysiol. 107, 2096-2108. https://doi.org/10.1152/jn.00931.2011 (2012).

43. Nitabach, M. N. et al. Electrical hyperexcitation of lateral ventral pacemaker neurons desynchronizes downstream circadian oscillators in the fly circadian circuit and induces multiple behavioral periods. J. Neurosci. 26, 479-489. https://doi.org/10.1523/jneur osci.3915-05.2006 (2006).

44. Cao, G. \& Nitabach, M. N. Circadian control of membrane excitability in Drosophila melanogaster lateral ventral clock neurons. J. Neurosci. 28, 6493-6501. https://doi.org/10.1523/jneurosci.1503-08.2008 (2008).

45. Fernández, M. P., Berni, J. \& Ceriani, M. F. Circadian remodeling of neuronal circuits involved in rhythmic behavior. PLoS Biol. 6, e69. https://doi.org/10.1371/journal.pbio.0060069 (2008).

46. Park, J. H. et al. Differential regulation of circadian pacemaker output by separate clock genes in Drosophila. Proc. Natl. Acad. Sci. 97, 3608. https://doi.org/10.1073/pnas.97.7.3608 (2000).

47. Martelli, F. et al. Low doses of the neonicotinoid insecticide imidacloprid induce ROS triggering neurological and metabolic impairments in Drosophila. Proc. Natl. Acad. Sci. 117(41), 25840-25850. https://doi.org/10.1073/pnas.2011828117 (2020).

48. Numata, H., Miyazaki, Y. \& Ikeno, T. Common features in diverse insect clocks. Zool. Lett. 1, 10. https://doi.org/10.1186/s4085 1-014-0003-y (2015).

49. Farris, S. \& Sinakevitch, I. Development and evolution of the insect mushroom bodies: towards the understanding of conserved developmental mechanisms in a higher brain center. Arthropod Struct. Dev. 32, 79-101. https://doi.org/10.1016/S1467 -8039(03)00009-4 (2003).

50. Jones, A. K. \& Sattelle, D. B. In Insect Nicotinic Acetylcholine Receptors (ed Thany, S. H.) 25-43 (Springer New York, 2010).

51. Homem, R. A. et al. Evolutionary trade-offs of insecticide resistance-the fitness costs associated with target-site mutations in the nAChR of Drosophila melanogaster. Mol. Ecol. 29, 2661-2675. https://doi.org/10.1111/mec.15503 (2020).

52. Blacquiere, T., Smagghe, G., van Gestel, C. A. \& Mommaerts, V. Neonicotinoids in bees: a review on concentrations, side-effects and risk assessment. Ecotoxicology 21, 973-992. https://doi.org/10.1007/s10646-012-0863-x (2012).

53. Stanley, D. A. \& Raine, N. E. Bumblebee colony development following chronic exposure to field-realistic levels of the neonicotinoid pesticide thiamethoxam under laboratory conditions. Sci. Rep. 7, 8005. https://doi.org/10.1038/s41598-017-08752-x (2017).

54. Whitehorn, P. R., O'Connor, S., Wackers, F. L. \& Goulson, D. Neonicotinoid pesticide reduces bumble bee colony growth and queen production. Science 336, 351. https://doi.org/10.1126/science.1215025 (2012).

55. Williamson, S. M., Willis, S. J. \& Wright, G. A. Exposure to neonicotinoids influences the motor function of adult worker honeybees. Ecotoxicology 23, 1409-1418. https://doi.org/10.1007/s10646-014-1283-x (2014).

56. Wright, G. A., Softley, S. \& Earnshaw, H. Low doses of neonicotinoid pesticides in food rewards impair short-term olfactory memory in foraging-age honeybees. Sci. Rep. 5, 15322. https://doi.org/10.1038/srep15322 (2015).

57. Malik, B. R. \& Hodge, J. J. Drosophila adult olfactory shock learning. J. Vis. Exp. 90, 50107. https://doi.org/10.3791/50107 (2014).

58. Hodge, J. J. \& Stanewsky, R. Function of the Shaw potassium channel within the Drosophila circadian clock. PLoS ONE 3, e2274e2274. https://doi.org/10.1371/journal.pone.0002274 (2008).

59. Moffat, C. et al. Neonicotinoids target distinct nicotinic acetylcholine receptors and neurons, leading to differential risks to bumblebees. Sci. Rep. 6, 24764. https://doi.org/10.1038/srep24764 (2016).

60. Busto, G. U., Cervantes-Sandoval, I. \& Davis, R. L. Olfactory learning in Drosophila. Physiology 25, 338-346. https://doi. org/10.1152/physiol.00026.2010 (2010).

61. Lyons, L. C. \& Roman, G. Circadian modulation of short-term memory in Drosophila. Learn. Mem. 16, 19-27. https://doi. org/10.1101/lm.1146009 (2009).

62. Depetris-Chauvin, A. et al. Adult-specific electrical silencing of pacemaker neurons uncouples the molecular oscillator from circadian outputs. Curr. Biol. 21, 1783-1793. https://doi.org/10.1016/j.cub.2011.09.027 (2011).

63. Baz, E.-S., Wei, H., Grosshans, J. \& Stengl, M. Calcium responses of circadian pacemaker neurons of the cockroach Rhyparobia maderae to acetylcholine and histamine. J. Comp. Physiol. A. 199, 365-374. https://doi.org/10.1007/s00359-013-0800-3 (2013).

64. Sheeba, V. et al. Large ventral lateral neurons modulate arousal and sleep in Drosophila. Curr. Biol. 18, 1537-1545. https://doi. org/10.1016/j.cub.2008.08.033 (2008).

65. Thany, S. H. Insect Nicotinic Acetylcholine Receptors (Springer, New York, 2011).

66. Gill, R. J. \& Raine, N. E. Chronic impairment of bumblebee natural foraging behaviour induced by sublethal pesticide exposure. Funct. Ecol. 28, 1459-1471. https://doi.org/10.1111/1365-2435.12292 (2014).

67. Bloch, G., Bar-Shai, N., Cytter, Y. \& Green, R. Time is honey: circadian clocks of bees and flowers and how their interactions may influence ecological communities. Phil. Trans. R. Soc. B 372, 20160256. https://doi.org/10.1098/rstb.2016.0256 (2017).

68. van Alphen, B., Yap, M. H. W., Kirszenblat, L., Kottler, B. \& van Swinderen, B. A dynamic deep sleep stage in Drosophila. J. Neurosci. 33, 6917. https://doi.org/10.1523/JNEUROSCI.0061-13.2013 (2013).

69. Buhl, E., Higham, J. P. \& Hodge, J. J. L. Alzheimer's disease-associated tau alters Drosophila circadian activity, sleep and clock neuron electrophysiology. Neurobiol. Dis. 130, 104507. https://doi.org/10.1016/j.nbd.2019.104507 (2019). 
70. Levine, J. D., Funes, P., Dowse, H. B. \& Hall, J. C. Signal analysis of behavioral and molecular cycles. BMC Neurosci. 3, 1. https:// doi.org/10.1186/1471-2202-3-1 (2002).

71. Faville, R., Kottler, B., Goodhill, G. J., Shaw, P. J. \& van Swinderen, B. How deeply does your mutant sleep? Probing arousal to better understand sleep defects in Drosophila. Sci. Rep. 5, 8454. https://doi.org/10.1038/srep08454 (2015).

72. Donelson, N. C. et al. High-resolution positional tracking for long-term analysis of Drosophila sleep and locomotion using the "tracker" program. PLoS ONE 7, e37250. https://doi.org/10.1371/journal.pone.0037250 (2012).

73. Schindelin, J. et al. Fiji: an open-source platform for biological-image analysis. Nat. Methods 9,676 . https://doi.org/10.1038/nmeth $2019(2012)$

\section{Acknowledgements}

We thank Drs Ralph Stanewsky, University of Münster, Germany and Scott Waddell, University of Oxford, UK for providing flies. We thank Drs Edgar Buhl, Stephen Montgomery and Herman Wijnen for providing comments on the manuscript and acknowledge the Wolfson Bioimaging facilities at University of Bristol. This work was supported by a BBSRC studentship BB/J014400/1, Leverhulme Project Grant RPG-2016-318 and Alzheimer's Research UK Interdisciplinary Research Grant ARUK-IRG2019B-003 awarded to J.J.L.H and CONICYT-PCHA/ Doctorado Nacional/2016-21161611 studentship to S.H.

\section{Author contributions}

J.J.L.H., K.T. and S.A.R. designed the study. K.T. performed and analysed the circadian and sleep assays, immunohistochemistry work, climbing and longevity and lethality assays. S.H. and J.J.L.H. carried out the learning and memory assays. B.Z carried out the qPCR assays. K.T. and J.J.L.H. wrote the paper with input from S.A.R. and S.H. The project was supervised by J.J.L.H. and S.A.R., who secured funding and edited the manuscript.

\section{Competing interests}

The authors declare no competing interests.

\section{Additional information}

Supplementary Information The online version contains supplementary material available at https://doi. org/10.1038/s41598-021-81548-2.

Correspondence and requests for materials should be addressed to J.J.L.H.

Reprints and permissions information is available at www.nature.com/reprints.

Publisher's note Springer Nature remains neutral with regard to jurisdictional claims in published maps and institutional affiliations.

(c) (i) Open Access This article is licensed under a Creative Commons Attribution 4.0 International License, which permits use, sharing, adaptation, distribution and reproduction in any medium or format, as long as you give appropriate credit to the original author(s) and the source, provide a link to the Creative Commons licence, and indicate if changes were made. The images or other third party material in this article are included in the article's Creative Commons licence, unless indicated otherwise in a credit line to the material. If material is not included in the article's Creative Commons licence and your intended use is not permitted by statutory regulation or exceeds the permitted use, you will need to obtain permission directly from the copyright holder. To view a copy of this licence, visit http://creativecommons.org/licenses/by/4.0/.

(c) The Author(s) 2021 